\title{
Randomized controlled trial of mibampator for behavioral and psychological symptoms of dementia: comments on the trial and thoughts for future studies
}

Behavioral and psychological symptoms of dementia (BPSD) frequently arise in people with Alzheimer's disease (AD) and other dementias. They cause significant distress and confer risk to the person and others, in addition to presenting a complex clinical challenge for treatment (Ballard et al., 2009b). There is good evidence for the value of first-line management strategies such as psychological interventions and treatment of concurrent medical conditions, particularly pain, which are known to be effective (Ballard et al., 2009b). However, there are limited pharmacological treatment options for severe aggression, which causes significant risk, and for other severe BPSD which do not respond to first-line approaches. The only pharmacological intervention with an adequate evidence base is the prescription of atypical antipsychotics, where 18 placebo-controlled trials have evaluated the effect of treatment over 6-12 weeks. The literature indicates modest but significant benefits in the treatment of aggression and psychosis with risperidone and aripiprazole (Cohen's $d$ standardized effect size of 0.2 ), uncertain benefits with olanzapine, and no benefits with quetiapine (Ballard and Howard, 2006; Schneider et al., 2006a; Ballard et al., 2009b; Corbett et al., 2012). Unfortunately, the benefits of longer term prescribing are more limited (Schneider et al., 2006b; Ballard et al., 2008) and there have been increasing concerns regarding the potential for serious adverse outcomes, including accelerated cognitive decline, stroke, and death (Schneider et al., 2006b; Ballard et al., 2009a). There is therefore an urgent imperative to identify more effective pharmacological treatments for severe BPSD which have a better safety profile, particularly for long-term treatment and prophylaxis. Despite this urgency, there has been very little effort toward developing or evaluating potential novel therapies for the treatment of key symptoms such as aggression, psychosis, restlessness, and apathy.

Several pharmacological alternatives have been suggested. Small preliminary trials have indicated that carbamazepine (Olin et al., 2001; Tariot et al., 1998; Ballard et al., 2009b), citalopram (Pollock et al., 2007; Ballard et al., 2009b), and prazosin
(Wang et al., 2009; Corbett et al., 2012) may confer benefit in the acute treatment of aggression or psychosis, and some possible longer term benefits for the prophylaxis of these symptoms with memantine (Ballard et al., 2009b). However, other postulated treatments such as sodium valproate and trazodone have not performed well in randomized controlled trial (RCTs) (Ballard et al., 2009b) and both cholinesterase inhibitors and memantine failed to improve agitation in RCTs over 12 weeks (Howard et al., 2007; Fox et al., 2012). Whilst there are some potentially promising candidates for further clinical trials, progress has been extremely slow. Disappointingly, there has been very little development work based on understanding of the biological mechanisms of key symptoms. The value of this approach has been highlighted by recent encouraging preliminary evidence for prazosin (Wang et al., 2009), with human autopsy studies indicating the relationship between altered adrenoceptors and agitation or aggression in people with AD (Sharp et al., 2007). The biological basis of other BPSD is likely to be different and involves separate mechanistic pathways. For example, there is no substantive evidence linking delusions to alterations in serotonergic or adrenergic function, but several post-mortem studies do indicate an association between delusions and upregulation of post-synaptic muscarinic receptors in the context of neurodegenerative dementias (Ballard et al., 2000; Teaktong et al., 2005). This is consistent with an RCT of the muscarinic agonist xanomeline in AD patients which reported improvements in delusions (Bodick et al., 1997). This preliminary body of evidence does provide support for the potential value of experimental medicine principles in identifying new therapies for BPSD, but so far there has been no systematic effort to identify potential agents for repurposing as BPSD treatments and there has been even less focus on developing novel pharmacological treatments.

In this edition of International Psychogeriatrics, Trepacz and colleagues (Trepacz et al., 2013) report an RCT of the novel glutamatergic $\alpha$-amino-3-hydroxy-5-methyl-4-isoxazolepropionic acid (AMPA) receptor modulator mibampator as a potential treatment for BPSD in AD. The 
AMPA receptor plays a role in the regulation of the glutamatergic system, which is known to be damaged in people with AD. The RCT is well designed and adequately powered, comparing mibampator to placebo in 132 outpatients with clinically significant agitation and/or aggression in the context of $\mathrm{AD}$ of moderate severity over 12 weeks. The trial unfortunately demonstrated no efficacy in treating agitation, aggression, or other neuropsychiatric symptoms, but nevertheless should be applauded as an excellent example of evaluating a novel therapeutic agent for the treatment of key BPSD. Many more such trials are urgently needed but it is important to also consider potential reasons why the trial was unsuccessful in order to strengthen the design of future studies. Although the study followed a well-established design, there are several key points within the trial that should be noted, including the use of concurrent cholinesterase inhibitors and memantine, and the use of other psychotropic medication. Both cholinesterase inhibitors and memantine impact on the glutamatergic systems and it is therefore possible that this activity may have confounded the outcome. Another important point is the high placebo response rate observed in the trial, which is consistently reported in trials of this nature within this field. In order to ameliorate this effect, an improved design might have been the inclusion of an initial lead-in stage or simple non-pharmacological intervention to filter out placebo responders prior to randomization.

If not as a consequence of methodology, why else might mibampator have been ineffective in the current RCT? The rationale for taking mibampator forward as a treatment for agitation and aggression was based on a phase II trial of mibampator for cognition, which did not demonstrate significant benefits in terms of cognition, but did result in improvement in key BPSD. Whilst this seems very reasonable as a potential rationale for taking a treatment forward to a further trial, there must always be a degree of caution with respect to overinterpreting secondary outcome measures from an RCT in the context of multiple outcomes. Whilst the statistical rationale is clear, the root of the lack of effect may be the absence of a plausible biological rationale linking mibampator to agitation, aggression, or other key BPSD. Mibampator is an AMPA receptor potentiator, which plays a role in the regulation of the glutamatergic system. The AMPA system also has important functions in the regulation of synapses, synaptic regeneration, and neuroprotection and is therefore a good therapeutic target for treatments aiming to improve cognition and function or alter disease progression. There is however less of a clear pathway linking the
AMPA system to agitation, aggression, or other BPSD.

In summary, we urgently need more therapeutic trials evaluating novel pharmacological treatments for agitation, aggression, psychosis, and other BPSD in order to improve the treatment of these common and distressing symptoms which significantly impact upon the lives of people with $\mathrm{AD}$ and those caring for them. New treatments will be key in avoiding an ongoing over-reliance of the use of antipsychotic agents as they are currently the only evidence-based pharmacological therapy. There are some methodological issues which could perhaps be usefully addressed by an expert consensus to optimize trial design but, most importantly, it is imperative that we have a strong emphasis on biological mechanisms and plausibility as a key component of further treatment development in this area. We already have good biological targets for repositioned and novel therapeutics, and preliminary evidence of efficacy for some candidate therapies. Further development and evaluation of the best candidates is an urgent treatment priority.

\section{Conflict of interest}

Clive Ballard has received research funding and consultancy/speaking honoraria from Lundbeck pharmaceutical company, speaking and consultancy honoraria from Bristol Myer Squibb pharmaceutical company, research funding, consultancy and speaking honoraria from Acadia pharmaceutical company.

Paul Francis has received speaker bureau honoraria, advisory board membership and research funding from Lundbeck pharmaceutical company, expert witness testimony in court cases involving Novartis and Janssen Alzheimer Immunotherapy.

Anne Corbett has received speaking/consultancy honoraria for Lundbeck, Novartis, Bial, and Acadia pharmaceutical companies.

\section{Clive Ballard, Paul Francis and \\ ANNE CORBETT \\ Institute of Psychiatry, Wolfson Centre for Age-Related Diseases, King's College London, London, UK \\ Email: clive.ballard@kcl.ac.uk}

\section{References}

Ballard, C. and Howard, R. (2006). Neuroleptic drugs in dementia: benefits and harm. Nature Reviews Neuroscience, 7, 492-500.

Ballard, C. et al. (2000). Delusions associated with elevated muscarinic binding in dementia with Lewy bodies. Annals of Neurology, 48, 868-876. 
Ballard, C. et al. (2008). A randomized, blinded, placebo-controlled trial in dementia patients continuing or stopping neuroleptics (the DART-AD trial). PLoS Medicine, 5,76 .

Ballard, C. et al. (2009a). The dementia antipsychotic withdrawal trial (DART-AD): long-term follow-up of a randomised placebo-controlled trial. Lancet Neurology, 8, 151-157.

Ballard, C. G. et al. (2009b). Management of agitation and aggression associated with Alzheimer disease. Nature Reviews Neuroscience, 5, 245-255.

Bodick, N. C. et al. (1997). Effects of xanomeline, a selective muscarinic receptor agonist, on cognitive function and behavioral symptoms in Alzheimer disease. Archives of Neurology, 54, 465-473.

Corbett, A., Smith, J., Creese, B. and Ballard, C. (2012). Treatment of behavioral and psychological symptoms of Alzheimer's disease. Current Treatment Option in Neurology, $14,113-125$.

Fox, C. et al. (2012). Efficacy of memantine for agitation in Alzheimer's dementia: a randomised double-blind placebo controlled trial. PLoS One, 7, e35185.

Howard, R. J. et al. (2007). Donepezil for the treatment of agitation in Alzheimer's disease. New England fournal of Medicine, 357, 1382-1392.

Olin, J. T., Fox, L. S., Pawluczyk, S., Taggart, N. A. and Schneider, L. S. (2001). A pilot randomized trial of carbamazepine for behavioral symptoms in treatment-resistant outpatients with Alzheimer disease. American Fournal of Geriatric Psychiatry, 9, 400-405.

Pollock, B. G. et al. (2007). A double-blind comparison of citalopram and risperidone for the treatment of behavioral and psychotic symptoms associated with dementia. American fournal of Geriatric Psychiatry, 15, 942-952.

Schneider, L. S., Dagerman, K. and Insel, P. S. (2006a). Efficacy and adverse effects of atypical antipsychotics for dementia: meta-analysis of randomized, placebo-controlled trials. American fournal of Geriatric Psychiatry, 14, 191210.

Schneider, L. S. et al. (2006b). Effectiveness of atypical antipsychotic drugs in patients with Alzheimer's disease. New England Fournal of Medicine, 355, 1525-1538.

Sharp, S. I., Ballard, C. G., Chen, C. P. and Francis, P. T. (2007). Aggressive behavior and neuroleptic medication are associated with increased number of alpha1-adrenoceptors in patients with Alzheimer disease. American fournal of Geriatric Psychiatry, 15, 435-437.

Tariot, P. N. et al. (1998). Efficacy and tolerability of carbamazepine for agitation and aggression in dementia. American fournal of Geriatric Psychiatry, 155, 54-61.

Teaktong, T., Piggott, M. A., Mckeith, I. G., Perry, R. H., Ballard, C. G. and Perry, E. K. (2005). Muscarinic $\mathrm{M} 2$ and M4 receptors in anterior cingulate cortex: relation to neuropsychiatric symptoms in dementia with Lewy bodies. Behavioural Brain Research, 161, 299-305.

Trzepacz, P. T. et al. (2013). Mibampator (LY451395) randomized clinical trial for agitation/aggression in Alzheimer's disease. International Psychogeriatrics, 25, 707-719.

Wang, L. Y. et al. (2009). Prazosin for the treatment of behavioral symptoms in patients with Alzheimer disease with agitation and aggression. American fournal of Geriatric Psychiatry, 17, 744-751. 